\title{
Digital Games Research: A Survey Study on an Emerging Field and Its Prevalent Debates
}

Thorsten Quandt ${ }^{1}$, Jan Van Looy ${ }^{2}$, Jens Vogelgesang ${ }^{3}$, Malte Elson ${ }^{4}$, James D. Ivory ${ }^{5}$, Mia Consalvo ${ }^{6}$, \& Frans Mäyrä7

1 Department of Communication, University of Münster, 48143 Münster, Germany 2 Research Group for Media and ICT, iMinds \& Ghent University, Ghent 9000, Belgium 3 Institute of Communication Studies, University Hohenheim, 70599 Stuttgart, Germany

4 Educational Psychology Research Group, Ruhr University Bochum, 44801 Bochum, Germany

5 Department of Communication, Virginia Tech, Blacksburg, VA 24061, USA

6 Department of Communication Studies, Concordia University, Montreal, Canada

7 School of Information Sciences, University of Tampere, Tampere, Finland

Digital games have become a popular form of media entertainment. However, it remains unclear whether a canon of accepted knowledge and research practices has emerged that may define an independent field of research. This study is a collaborative effort to analyze the outlines of digital games research (DGR) through a survey among the membership of 3 institutionalized structures focusing on the study of digital games (International Communication Association Game Studies Interest Group, European Communication Research and Education Association Temporary Working Group DGR, and Digital Games Research Association). The study reveals relatively homogeneous viewpoints among games researchers, even regarding controversial aspects of digital games. It mirrors the mainstream scholarly views on contentious issues of a recently emerged field within communication studies.

Keywords: Digital Games Research, Survey, Disciplinary Development, Science of Knowledge, ICA, DiGRA, ECREA.

doi:10.1111/jcom.12182

In the past few decades, digital gaming has developed from a marginal spare-time activity of a small societal group into a driving force of the entertainment industry and part of the mainstream culture of modern societies. Recent international studies also reveal that digital games' usage numbers have grown considerably, and that digital games are now played by large groups in society (Quandt, Chen, Mäyrä, \& Van Looy, 2014) beyond initial core audiences that were stereotypically associated with

Corresponding author: Thorsten Quandt; e-mail: thorsten.quandt@uni-muenster.de 
male teens (Griffiths, Davies, \& Chappell, 2003): The majority of users are adults, and while there are still more male than female players, the gap between the two groups is shrinking with a recent report by Entertainment Software Association (2014) indicating that $48 \%$ of Americans who play games are female. Furthermore, numerous studies discuss the potential impact of digital games on their users, from the effects of violent content to behavioral addiction, from changes in the social life of gamers to learning effects (Connolly, Boyle, MacArthur, Hainey, \& Boyle, 2012; Ivory, 2013). In that respect, digital games can be considered a highly relevant topic to communication studies.

However, the rise of academic interest in digital games is a relatively recent development, especially when compared to the medium's historic roots that can be traced back more than 60 years to the earliest computer game "Noughts and Crosses" (or "OXO") - developed by Alexander S. Douglas in 1952. It was not until the late 1990s, however, that social sciences and humanities "discovered" digital games as a respectable research area (Mäyrä, Van Looy, \& Quandt, 2013). Several strands of research were competing in the early phase of game studies: Williams, for example, noticed a "methodological divide in game research" (Williams, 2005, p. 1) that mirrored two approaches-one being based in social sciences and aiming "to understand the effects of games on users," and the other one being based in humanities and seeking "to understand the meaning and context of games."

Yet, even within these larger movements, various perspectives emerged (cf. Ivory, 2013; Van Looy, 2010). One of the most prominent debates in the humanistic tradition concerned that between narrativists, who primarily saw games as a new form of storytelling, and ludologists, who resisted this view and called for a radically new approach focusing on game mechanics (Pearce, 2005). In the social sciences, researchers fiercely discussed the impact of violent content of digital games, with some researchers claiming undisputable proof of strong effects (Anderson et al., 2010), and others finding only moderate or nonexistent effects (Ferguson, 2007) or interpreting the effects as largely unproblematic (Sherry, 2001). This debate in the social sciences is ongoing, leading to a situation whereby some researchers have declared a final "consensus" (Bushman, Gollwitzer, \& Cruz, 2014), whereas others have publicly disputed the legitimacy of earlier claims (Consortium of Scholars, 2013).

Despite these debates and sometimes adverse viewpoints, one can observe a growing organization of research efforts, as well as signs of formal institutionalization of game research as a distinct field within the social sciences and humanities, and communication studies in particular. ${ }^{1}$ The first steps toward an organizationally more defined field were taken in the early 2000s, with the establishment of dedicated journals (such as Game Studies in 2001 and Games and Culture in 2006) and interested academics discussing game topics in specialized blogs (such as Terra Nova, which was established in 2003). Many of these researchers felt that there was no place for the analysis of games in their home disciplines, whereas other academics argued that the study of games deserved its own discipline: 
Today we have the possibility to build a new field. ( ... ) Of course, games should also be studied within existing fields and departments, such as Media Studies, Sociology, and English, to name a few. But games are too important to be left to these fields. (And they did have thirty years in which they did nothing!) ( ... (Game Studies) should exist as an independent academic structure, because it cannot be reduced to any of the above. (Aarseth, 2001)

Indeed, an independent association, the Digital Games Research Association (DiGRA), was established in 2003, uniting researchers from various disciplinary backgrounds as well as practitioners interested in the research of digital games. However, research on digital games was also gradually picked up by existing disciplines. Within communication studies, interest groups were established to channel the interest of researchers and provide a home to the study of games: In the International Communication Association (ICA), a Game Studies Interest Group was established in 2005 and became a division in 2015; and in 2011, a Digital Games Temporary Working Group was set up in the European Communication Research and Education Association (ECREA), which became a section in 2015. Overall, there has been considerable growth of research on digital games, and a consolidation of a community with a shared interest in social interaction and communication in games. This can be exemplified by the scientific output published, even in general-interest journals that are not dedicated to research on games. Although there were only occasional publications on digital games in the leading communication and media psychology journals some 10 years ago, there is now a constant stream of findings (Table 1).

Although today digital games have been accepted as a popular form of interactive media entertainment, and, concomitantly, a topic for communication research, it remains unclear whether a canon of accepted knowledge, research practices, and dissemination channels has emerged, and whether digital games research (DGR) is truly accepted as an independent field (for a related discussion on Internet research, see Baym, 2005).

Studying this emerging field of DGR within communication studies is a worthwhile effort: As this is still a field "under construction," one will not only learn about the evolution of this specific field but also learn about the initial development of fields and research ecologies in the social sciences in a more general sense. Arguably, changes of this type and magnitude - whereby the emergence of a new medium elicits interest from a broad range of fields and (sub)disciplines, provoking power struggles, but also facilitating cross-fertilization - do not regularly occur.

Although there are various historic and partially anecdotal descriptions of the emergence and differentiation of game studies within the humanities and social sciences (Mäyrä, 2008; Mortensen, 2009), no systematic empirical research has yet been conducted on the status of digital games within communication studies and related disciplines. This study is a unique collaborative effort to describe and analyze the field of DGR and its links to media and communication studies through a survey among the membership of three highly relevant institutionalized structures for games research, that is, DiGRA, the ICA Game Studies Interest Group, and the Temporary 


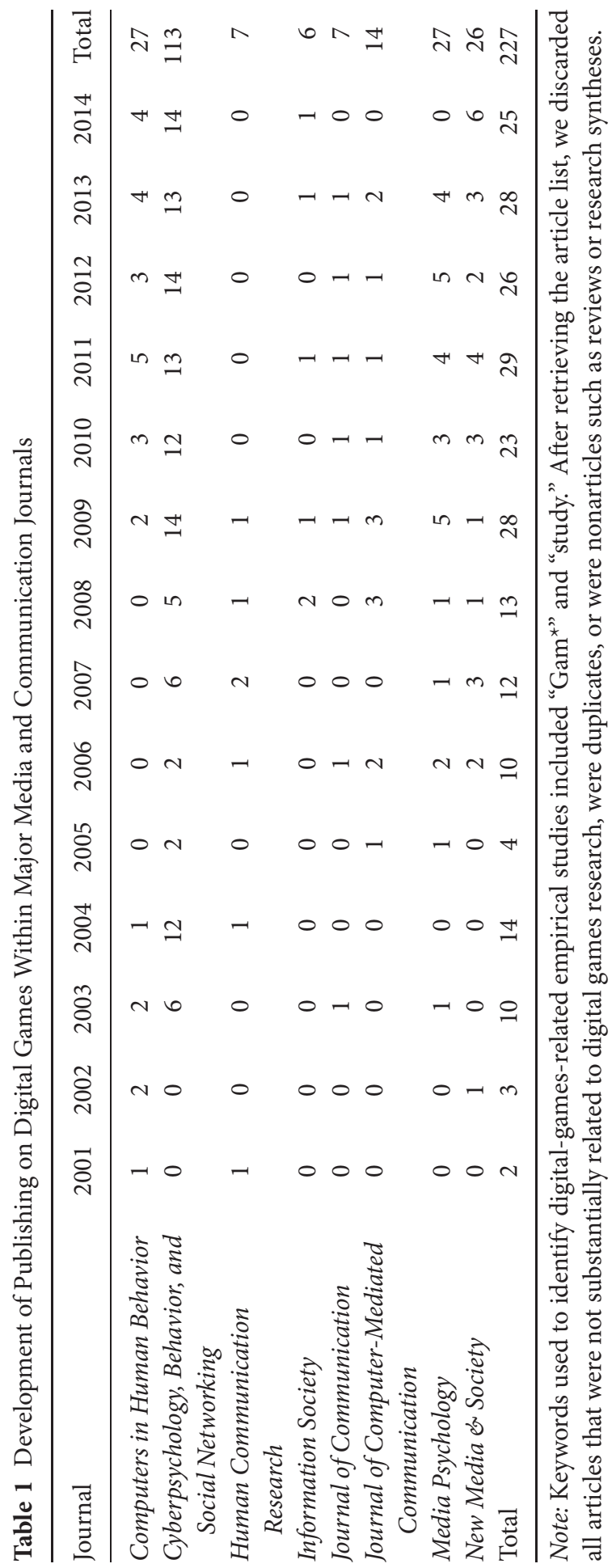


Working Group Digital Games Research of ECREA. Although the ICA and ECREA groups are fully located inside communication studies, DiGRA, as a transdisciplinary association within social sciences and humanities, is more open but still has close ties to the analysis of social interaction, communication, and media.

On a primary level, the aim of this study is a description of the status quo in the field, as reconstructed based on the views and opinions of research practitioners. Therefore, we inquire into who the members of this field are, where they come from, and what their opinions about games research and the development of the field are. On a secondary level, this study is interested in the question of whether there are different disciplinary viewpoints within this field and to what extent these are represented in some of the ongoing debates, which may explain tension as well as the propelling forces that push the development of the field. In that respect, this study is informed by the science of knowledge, which is interested in the social processes that lead to the emergence of new ways of thinking and new knowledge.

\section{New topics, new groups, new science? Approaches to the science of knowledge}

As noted above, we are focusing on the development of DGR within the social sciences and humanities, and communication studies in particular. Analyzing the factors that influence and shape the development of knowledge is crucial not only for the understanding of such a field but also for the evolution of knowledge per se. The sociology of knowledge dates back to early works of Durkheim and Mauss (1901-1902), who were interested in the shaping of thought and language by the groups in which they are developed. Later, sociologists such as Mannheim (1929) focused on the sociology of knowledge, and turned this into a central research topic. Social constructivism (Berger \& Luckmann, 1966) notes that socialization is an important factor in perceiving interpretative schemes as objective reality. According to this approach, there is no "absolute" knowledge; rather, it is constructed in a social process both inside and outside of science. In a similar vein, Kuhn (1962) describes knowledge as being constructed in The Structure of Scientific Revolutions (1962). According to Kuhn, there are phases of "normal science" characterized by mere incremental changes and detailed work on existing concepts and ideas, and drastic "paradigm shifts" that more fundamentally alter the basic assumptions and perceptions in a given part of science. Whilst initially conceived with the natural sciences in mind, the work of Kuhn has also been applied to developments in the social sciences (e.g., Polsby, 1998). Radical macrolevel paradigm shifts are often preceded by phases of debate and struggle regarding what can be considered a legitimate topic within existing disciplines. Indeed, the debates about where to fit online topics and digitization - and more specifically, in this case, digital games - within disciplines like communication studies might be approached with such a conceptual framework.

A complementary perspective focuses on the meso level of groups and organizations in specific (sub)fields. Processes on this level can be understood as the 
organizational and personal basis for such macrolevel developments, as science does not happen in a void, but consists of the actions of individuals and groups in specific contexts. As noted by reference group theory (Hyman, 1942; Merton, 1957), humans use groups as comparisons, and these may serve as benchmarks for values, attitudes, behavior, and even thinking. According to Merton (1957), an individual does not necessarily have to be a member of such a reference group in order to position herself or himself according to group norms. Applied to academic knowledge production, this means that the notion of "acceptable" and even "desirable" knowledge is shaped through more or less visible groups that scientists relate to (Merton \& Zuckermann, 1973). Some of the most obvious ones are "disciplines" (that indicate membership through degrees and titles, among other means of belonging), more fluid "fields," and institutionalized groups like "associations" and their subsections, but also informal groups that form around an interest or specific mindset. In such groups, additional factors like age (Gingras, Larivière, Macaluso, \& Robitaille, 2008; Zuckerman \& Merton, 1973) and accumulated advantage (the so-called Matthew effect; see Merton, 1968) may also play a role in who has the most "impact" and is influential in the knowledge building processes. Accumulated advantage means that established scientists will receive more attention for their work, which will further enhance their reputation, whereas the work of unknown scholars will be largely ignored. Obviously, knowledge-shaping practices may support knowledge production by pronouncing novel or reputable ideas, but they may also carry an adverse effect in that existing structures can resist discoveries that do not follow the established patterns of the group (Barber, 1961). Overall, these findings support the notion of centripetal forces being at work within scientific disciplines in order to maintain coherence during phases of "normal" science.

\section{Disciplines and emerging fields: A heuristic-systematic model}

Following the basic argument about scientific disciplines and topical fields being reference contexts for scientific work and personal scientific careers, one can deduct several potential configurations of what happens when existing disciplines are confronted with a new phenomenon or approach (Figure 1).

When new phenomena (or new scientific approaches) emerge, they are positioned in relation to preexisting knowledge and approaches that are either part of the center or the periphery of a discipline. Typically, innovation will initially occur in the periphery rather than in the center of a discipline, as the center will try to integrate new phenomena on the basis of a canon of accepted knowledge, rules, and approaches (Lemaine, Macleod, Mulkay, \& Weingart, 1976). In other words, it will try to "normalize" that which is divergent. Peripheral parts of a discipline can be regarded as being more flexible and less consolidated, and there may also exist overlap with other disciplines. Therefore, although similar communities may develop within different disciplines around a certain phenomenon, they are at least partially still shaped by the already existing structures (research organizations, academic positions, etc.). It can be 


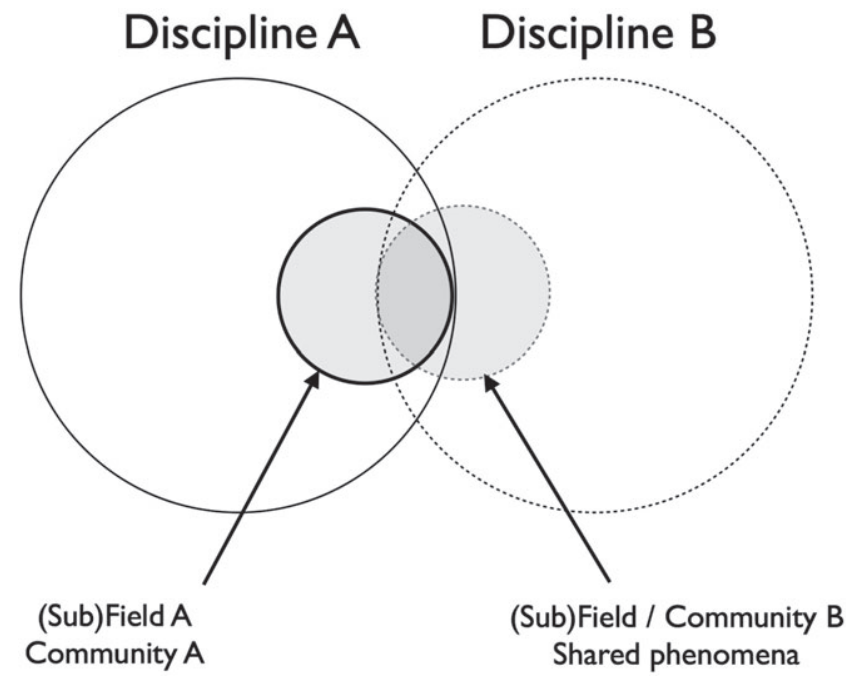

Figure 1 Emerging fields vis-à-vis existing disciplines.

argued that the disciplines as larger reference groups will also try to actively integrate the emerging fields and communities to reduce overlap and distinguish themselves from other disciplines in order to keep their own identity.

Applied to DGR, this means that the analysis of digital games as a new phenomenon can happen within disciplinary bound (sub)fields and communities and inbetween existing disciplines in topical fields located at the edges of different disciplines. Both perspectives are not mutually exclusive, but describe two ways of approaching the same development. Indeed, there is anecdotal evidence that both the development of a topical field that spans across disciplines and disciplinary subgroup differentiation are happening at the same time: The foundation of interest groups within communication associations and related social-scientific disciplines can be seen as a development toward discipline-bound groups, and even DiGRA as a transdisciplinary association is still operating very much within a shared field of social scientists with an interest in media and communication. On the other hand, there are other groups like ACM SIGCHI (a special interest group within the Association for Computing Machinery) or the IEEE Computational Intelligence Society that focus on digital games primarily from other disciplinary backgrounds (in this case, computing and engineering), with only limited overlap with the previously mentioned groups in terms of approaches and membership.

However, even within disciplines, different contexts of doing research may matter (Figure 2). When new phenomena are addressed or new approaches develop, this affects existing communities that revolve around related topics. These existing communities are most likely located near the center of the scientific community representing a discipline (as they are strongly associated with the established rules, norms, and knowledge). Between the novel community and the center, there might be some 


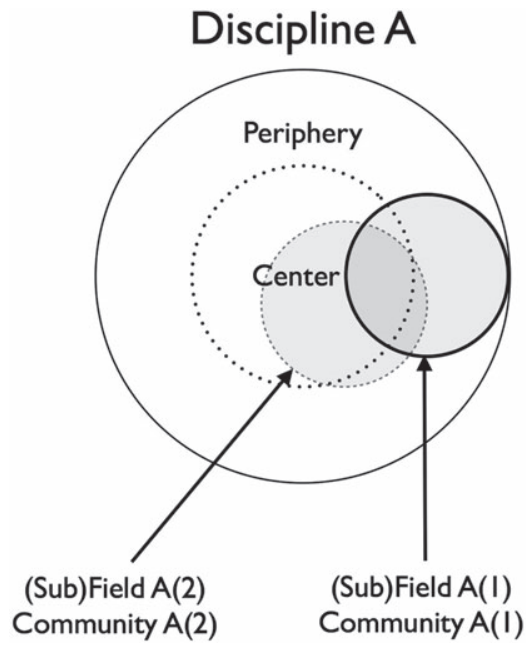

Figure 2 Emerging fields within an existing discipline.

topical overlap, which can lead to a situation in which discussions about a prerogative of interpretation regarding the phenomena may arise. Existing groups may claim that they sufficiently cover the new phenomenon (so that, for example, no formalized new structure for the new (sub)field is necessary).

Examples of this dynamic can be found in DGR as a field within communication studies as well. Debates on the relative independence of the topical field accompanied the establishment of working groups within academic societies (like ICA and ECREA), with existing divisions and sections being skeptical about the need for a new group. Furthermore, controversial aspects of digital gaming - namely the effects of violent content and addictive effects - were part of the debates between different groups within academic associations. In the ongoing debate on violent content, for example, reputation in specific reference groups (and, mediated by that, position and seniority) has been used as an argument for the validity of findings (Pollard Sacks, Bushman, \& Anderson, 2011), reflecting the struggle between center and periphery within the field. Often, this correlates with other group affiliations, sometimes rather informal ones (such as generational/age groups). Thus, differing perspectives may mirror underlying disciplinary demarcations and varying reference groups.

However, although these are more or less plausible assumptions on DGR as an academic field within communication and media studies, these assumptions have never been empirically assessed. In this study, we focus on the development from within the newly established groups and communities (i.e., the gray middle ground of model 1 , depicting the emerging field). Accordingly, we have conducted a survey of members of the most relevant digital games groups for said field (with the ICA and ECREA groups being fully integrated in the disciplinary boundaries, whereas DiGRA overlaps into other fields as well). Special attention has been given to discussions on controversial debates, following the argument above. 


\section{Research interest}

As noted above, this study is first of all interested in an empirical depiction of the emerging field of DGR. No comparable study exists to date, so the research questions are partially explorative in nature. However, previous literature (as discussed above) can give us some indication on factors of influence on the scholars in the field.

First and foremost, focusing on the academic (disciplinary) background self-perceptions, and attitudes of the researchers themselves, the study has a very basic research interest:

RQ1: What is the scientific background and personal profile of researchers in the field of DGR?

This RQ can be derived directly from the heuristic model. However, RQ1 is not as simple as it may seem, as even within the three groups that form the base population, very different disciplinary backgrounds may exist (i.e., media psychologists who are members of the ICA group, designers and computer scientists who are part of the ECREA working group, etc.), reflecting the topical diversity and the personal disciplinary heritage of lateral entrants.

Given the anecdotal evidence of disciplinary struggles and oppositional perspectives in games research, as discussed in the previous sections, we also suspect a difference in the perception of digital games as being harmful or beneficial, based on scholars' scientific socialization processes. It should be noted that these discussions are by no means representative of the rich work in the field. The effects discussion should merely be seen as an illustrative case for which past claims had been made regarding consensus within the field, that is, an interesting instance to evaluate a number of assumptions regarding the formation of the field. As there are conflicting depictions of the field so far, we propose an undirected RQ here:

RQ2: How are different viewpoints regarding harmful and beneficial effects of digital games distributed among digital games researchers in relation to disciplinary background?

Beyond belonging to a formal group as outlined by disciplinary affiliation, other differences between researchers might exist, denoting informal groups with similar self-definitions and attitudes toward the common research topic. We suspect that age and seniority may contribute to a more skeptical viewpoint toward the "new medium," in line with research that implies a more negative perspective toward new developments and changes among already established scholars (cf. Gingras et al., 2008; Zuckerman \& Merton, 1973). Therefore, we can hypothesize as follows:

H1: With a higher age and more seniority, digital games researchers will have a more negative view of the medium.

Furthermore, it seems to be plausible from the literature review and reference group theory that identification with a subject area may also contribute to a more positive attitude toward it (see also Turner, Oakes, Haslam, \& McGarty, 1994), and to a reduced belief in harmful effects. Such a strong identification may relate to the field, that is, whether the researcher defines her/himself as a games researcher, but also to 
the medium under analysis, that is, whether the researcher thinks of her/himself as being "a gamer."

H2a: If a scholar perceives her/himself as a digital games researcher, she/he will have a stronger belief in beneficial effects for society and perceive games as less harmful, when compared with other academics in the field.

H2b: If a scholar perceives her/himself as a gamer, she/he will have a stronger belief in beneficial effects for society and perceive games as less harmful, when compared with other academics in the field.

Identification is not to be confused with playing games, as "being a gamer" denotes being part of a specific gamer culture (De Grove \& Van Looy, 2015; Shaw, 2012). Playing games oneself may positively affect the perception of the medium as being beneficial, or reduce the belief in harmful effects, as it may contribute to the reduction of uncertainty and prejudices that may apply; on the other hand, it may also support previous skepticism, and therefore reduce the belief in beneficial effects or raise the belief in harmful effects. Furthermore, the amount of play may have an effect as well. As there are arguments for effects in both directions, no directed hypothesis will be formulated for gaming behavior.

RQ3: What kind of impact does the digital games researchers' gaming behavior (i.e. playing per se, and the volume of playing) have on the attitudes regarding beneficial or harmful effects of games?

Naturally, much more complex patterns of influence on the development of scientific knowledge and beliefs in the given field may exist. However, for this initial analysis, we deliberately limit the factors and effects, in order to produce baseline data for future studies.

\section{Methods}

\section{Participants}

Respondents were recruited in collaboration with the ICA's Game Studies Interest Group (IG) (now an ICA division), the ECREA's Temporary Working Group (TWG) DGR (now an ECREA section), and the DiGRA. A web survey link was distributed using online social media venues of the academic associations, as well as e-mail requests to their members in October 2012. At that time, the GAMESNETWORK mailing list of DiGRA comprised approximately 1,500 members, the ECREA TWG Facebook group had 180 members, and the ICA Game Studies IG had around 200 members. According to the log file of the web survey system, the introduction page had $n=792$ unique visitors. Incomplete questionnaires and respondents who did not correctly answer control questions (Meade \& Crais, 2012) were excluded from analysis, resulting in a sample of $n=544$ respondents. After exclusion of students $(n=35)$ and designers $(n=18)$ as ineligible and after deletion of respondents who refused to give personal information such as age or academic position $(n=8)$, the final sample analyzed in this study comprised $n=483$ respondents from 
41 countries. Males accounted for almost two thirds (64\%) of the sample. Mean age of the participants was 36.05 years $(S D=9.30)$, ranging from 19 to 79 years. Respondents were primarily working in Europe (56\%) and North America (33\%). The country with the highest number of respondents was the United States (27\%).

\section{Measures}

Respondents filled out an online survey featuring questions on their academic background, their gameplay behavior, their self-perception as scholars and as gamers, their attitudes regarding the subject area, and some items on sociodemographics. Items were reviewed by experts and pretested on a small scale $(N=11)$.

Academic background was gathered using a list of disciplines, with an open item for the "other" category. The answers to the open items were recoded into the broader categories where possible. Respondents were asked using dichotomous items as to whether they were teaching in the field of digital games and whether they actively played at all. We used two 5-point Likert items $(1=$ strongly disagree and $5=$ strongly agree) to measure self-perception as a digital games researcher and seeing oneself as a gamer. The volume of the respondents' own gaming behavior was retrieved in minutes per day. Finally, the questionnaire contained items on sociodemographics (age and gender; education was not considered, as this is a study in an academic context).

Regarding attitude toward digital games, respondents were asked to rate six items on a 5 -point Likert scale ( $1=$ strongly disagree and $5=$ strongly agree). To explore the dimensionality of the items and to address measurement error, we used a principal axis factor (PAF) analysis with oblimin rotation (i.e., factors are allowed to correlate). We used the Kaiser criterion (Eigenvalues $>1$ ), a scree plot, and the MAP criterion (Velicer, 1976) to evaluate the optimal number of factors. All three criteria suggested that a two-factor solution is appropriate. The two-factor solution accounted for $62 \%$ of the overall variance. The two-dimensional solution showed a simple structure (i.e., the structure matrix contained one high factor loading per factor for each item). The two factors were interpreted as (a) the belief that digital games can have beneficial effects and (b) the belief that digital games can have harmful effects.

\section{Belief that digital games can have beneficial effects}

Based on the following items, we computed a mean index $(\alpha=.72)$ : "Digital games can have beneficial effects on their users" $(M=4.34, S D=0.64)$, "the potential of using digital games in health is promising" $(M=4.17, S D=0.83)$, and "the potential of using digital games in education is promising" $(M=4.04, S D=0.81)$. The index variable subsequently served as one of two dependent variables in an analysis of covariance (ANCOVA) model.

\section{Belief that digital games can have harmful effects}

The second mean index $(\alpha=.65)$ was based on the following items: "Digital games can have harmful effects on their users" $(M=3.19, S D=1.02)$, "the addiction effects of digital games are a problem for society" $(M=2.61, S D=0.99)$, and "the effects of digital games on aggression behavior are a problem for society” $(M=2.18, S D=0.98)$. 


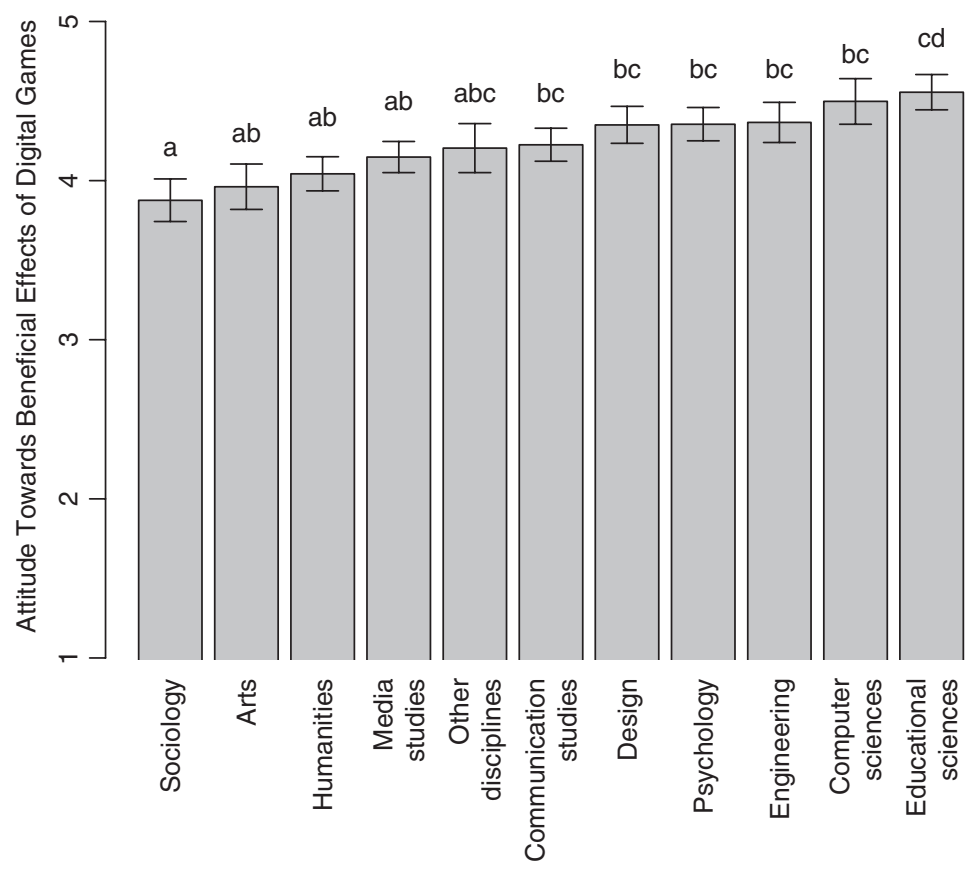

Figure 3 Estimated marginal means and 95\% confidence intervals of the game researchers' attitudes toward beneficial effects of digital games $(n=483)$.

Note: Error bars represent mean $\pm 1 S E$. Bars with different subscripts differ significantly at $p<.05$ using Šidák-adjusted multiple comparisons.

\section{Statistical analysis}

As we were interested in the attitudes and viewpoints of games researchers regarding beneficial and harmful effects of digital games, comparisons were made using ANCOVA. We estimated two separate general linear models (GLMs) with two mean indices as dependent variables. We used the GLM procedure with gender, academic discipline, and teaching experience as fixed factors and time gaming per day and self-perception measures as covariates. Following the recommendations by Field (2009) for ANCOVA, we used the Šidák (1967) adjustments for multiple comparisons (Figures 3 and 4). In addition, we report $\eta^{2}$ and $d$ as effect size estimates (Cohen, 1988; see Tables 3 and 4).

\section{Results}

Based on the findings, an initial impression of the overall group of researchers in the field emerges, answering RQ1. The respondents represented various fields of study such as Media Studies (18\%), Communication Studies (14\%), Psychology (12\%), Educational Science (11\%), Humanities (11\%), Design (9\%), Engineering (7\%), Sociology (6\%), the Arts (5\%), and Computer Sciences (4\%). Three percent of 


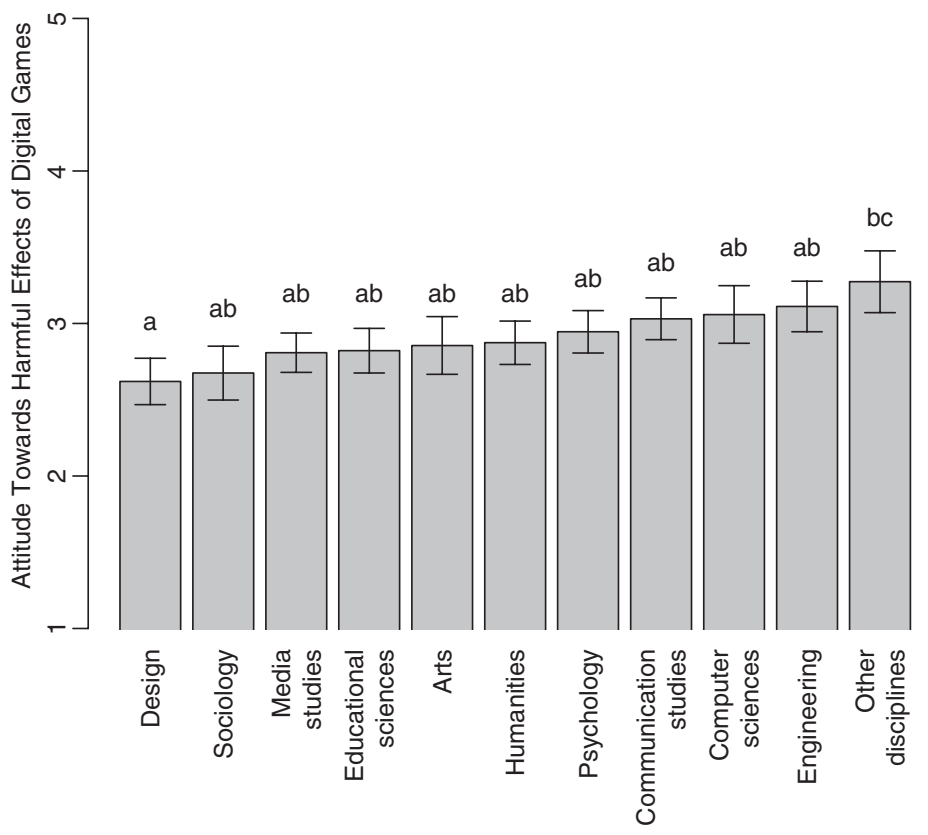

Figure 4 Academic disciplines of games researchers and their attitude toward harmful effects of digital games $(n=483)$.

Note: Error bars represent mean $\pm 1 S E$. Bars with different subscripts differ significantly at $p<.05$ using Šidák-adjusted multiple comparisons.

the respondents belonged to other academic disciplines. Thirty-four percent of the respondents held a professorship, 32\% were enrolled as $\mathrm{PhD}$ students, $20 \%$ worked as research staff, $10 \%$ as faculty, and $4 \%$ held other kinds of research positions. In line with Lemaine et al. (1976), who state that researchers succeeding in an emerging field are rather young, the age difference between professors $(M=41.06, S D=9.62)$ and PhD students $(M=30.55, S D=5.88)$ is relatively small. Eighty-one percent of all respondents considered themselves as digital games researchers. Sixty-five percent of all participants indicated teaching at least some courses dealing with digital games. The participants also reported playing 65.85 minutes $(S D=65.09)$ per day on average. Consistent with the nonacademic gaming population, male researchers indicated that they play more minutes per day on average than their female counterparts $\left(M_{\text {males }}=70.99, S D_{\text {males }}=68.36, M_{\text {females }}=56.72, S D_{\text {females }}=57.92, t(481)=-2.33\right.$, $p<.05, d=.21$ ).

As elaborated before, scholars dispute whether digital games have beneficial, harmful, or any effects on individual gamers or society as a whole. Table 2 shows that survey participants overall tended to agree that digital games could have beneficial effects $(M=4.18, S D=0.61)$. The view that playing digital games could have harmful effects is less accepted among games researchers $(M=2.66, S D=0.76)$. Interestingly, both attitudes are not significantly correlated, $r(483)=.08, p=.08, d=.16$. 


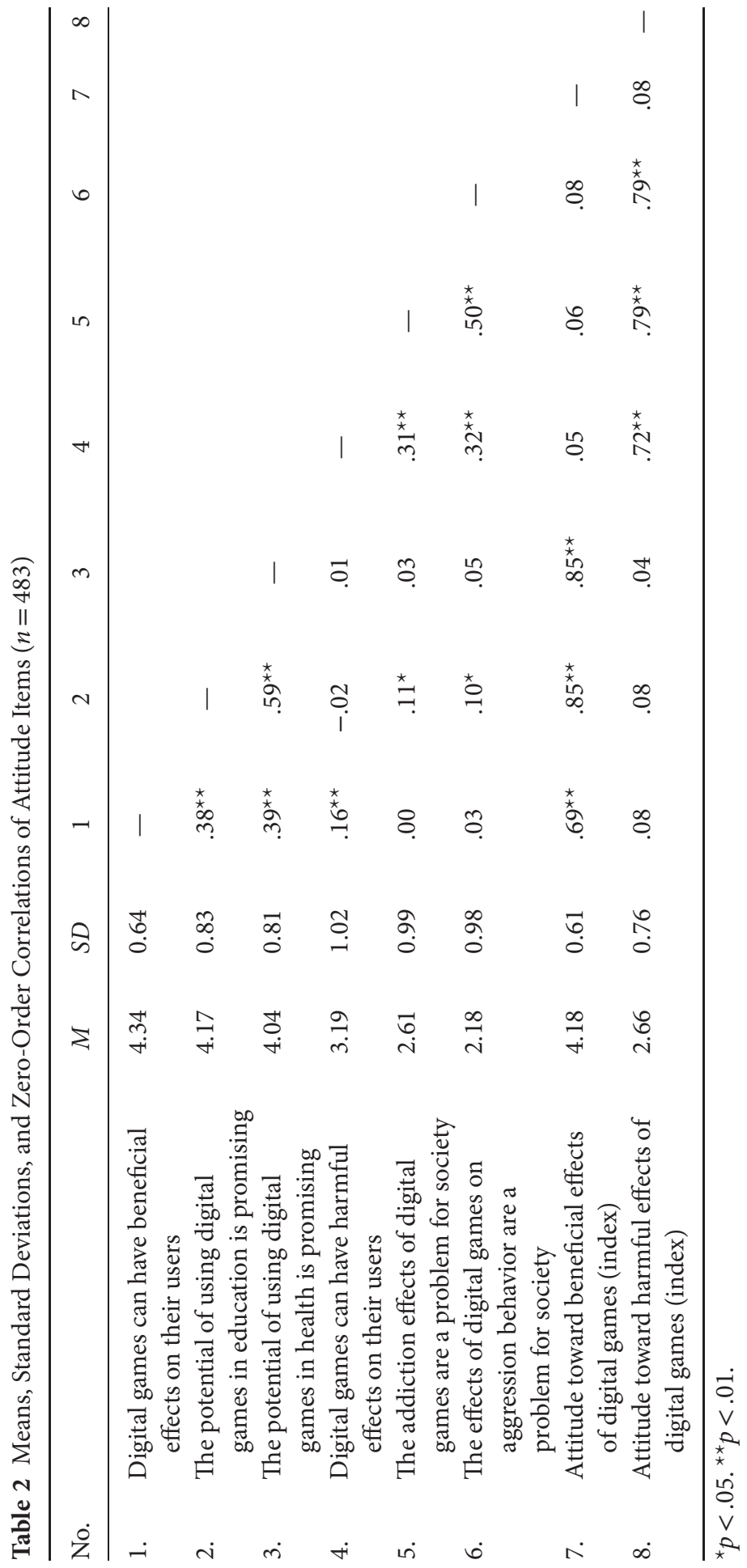


Table 3 Impact of Sociodemographics, Academic Background, Gaming Behavior, and Self-Perception of Digital Games Researchers on Attitude Toward Beneficial Effects of Digital Games $(n=483)$

\begin{tabular}{|c|c|c|c|c|c|}
\hline Source of Variance & $\begin{array}{l}\text { Sum of } \\
\text { Square }\end{array}$ & $d f$ & $F$ & $\eta^{2}$ & $d$ \\
\hline \multicolumn{6}{|l|}{ Sociodemographics } \\
\hline Age (years) & 1.908 & 1 & $6.063^{*}$ & 0.011 & 0.211 \\
\hline Gender & 1.122 & 1 & 3.534 & 0.006 & 0.155 \\
\hline \multicolumn{6}{|l|}{ Academic background } \\
\hline Teaching digital games (yes) & 3.566 & 1 & $11.333^{\star *}$ & 0.020 & 0.286 \\
\hline Discipline & 16.402 & 10 & $5.213^{* *}$ & 0.093 & 0.640 \\
\hline \multicolumn{6}{|l|}{ Gaming behavior } \\
\hline Time gaming per day (minutes) & 0.238 & 1 & 0.756 & 0.001 & 0.063 \\
\hline Never actively played digital games (yes) & 0.013 & 1 & 0.041 & 0.000 & 0.017 \\
\hline \multicolumn{6}{|l|}{ Self-perception } \\
\hline I see myself as a digital games researcher & 5.016 & 1 & $15.942^{\star *}$ & 0.028 & 0.339 \\
\hline I see myself as a gamer & 1.673 & 1 & $5.318 *$ & 0.009 & 0.191 \\
\hline Error & 146.299 & 465 & & & \\
\hline
\end{tabular}

Note: $R^{2}=.155$.

${ }^{*} p \leq .05 .{ }^{* *} p \leq .01$.

A more detailed analysis answers RQ2 and the various hypotheses: Table 3 shows the impact of sociodemographics, academic background, gaming behavior, and self-perception of the games researchers on their attitude toward beneficial effects of digital games $\left(R^{2}=.155\right)$. Detailed inspection of the GLM parameter estimates indicated that older respondents significantly agreed more that digital games could have beneficial effects, $F(1,465)=6.06, p<.05, d=0.21$. The beneficial effects attitude is dependent on the fields of study of the researchers, $F(10,465)=5.21, p<.01, d=0.64$ (Table 3). Multiple comparisons showed that researchers from sociology $(M=3.88)$ were most skeptical, whereas their peers from educational sciences $(M=4.56)$ had the most positive attitude toward potential for beneficial effects (Figure 3). Significant negative attitudes were also found among researchers of the Arts $(M=3.96)$ and Humanities $(M=4.04)$, whereas computer scientists $(M=4.50)$ had a more positive attitude. Communication scholars were among the disciplines that occupied the middle ground $(M=4.23)$. Researchers who do not teach digital games $(M=4.32)$ indicated a significantly stronger belief that digital games have beneficial effects than their colleagues who do teach digital games, $M=4.14 ; F(1,465)=11.33, p<.01$, $d=0.29$. Seeing oneself as a games researcher, $F(1,465)=15.94, p<.01, d=0.34$, and considering oneself as a gamer, $F(1,465)=5.32, p<.05, d=0.191$, were significantly correlated with the attitude that playing digital games is beneficial (partially supporting $\mathrm{H} 2 \mathrm{a}$ and $\mathrm{H} 2 \mathrm{~b}$ ). No effects of whether the scholars play games themselves or their own playing time were found (RQ3). 
Table 4 shows the effects of the predictor variables on the attitude toward potential harmful effects of digital games (RQ2; $R^{2}=.070$ ). Researchers' attitudes toward harmful effects were also dependent on their academic discipline, $F(10,465)=2.12$, $p<.05, d=0.42$ (Figure 4): Researchers coming from design $(M=2.62)$ were least likely to agree, whereas researchers coming from "other disciplines" $(M=3.27)$ were most likely to agree with the harmful effects position. However, all other multiple differences were not statistically significant. In addition to the disciplinary differences, we found a small effect addressing RQ3. Respondents who never actively played digital games $(M=3.10)$ were significantly more convinced that playing digital games can have harmful effects than those who once had played or still play $(M=2.67)$, $F(1,465)=4.254, p<.05, d=.20$. No significant effect could be found for sociodemographics (not supporting $\mathrm{H1}$ ), and the self-perception as digital games researcher or gamer (i.e. no support for $\mathrm{H} 2 \mathrm{a}$ and $\mathrm{H} 2 \mathrm{~b}$ ).

\section{Discussion}

This first survey of researchers in the emerging field of DGR draws a somewhat differentiated picture from previous anecdotal evidence in the literature. First of all, the academic background of digital games researchers in the groups under analysis (ECREA TWG, ICA IG, and DiGRA) is not as diverse as expected: There is a large group of communication and media scholars in the field, and more generally speaking, many of the researchers have a social-scientific background (with a notable group of psychologists taking part in the discussion). This may not come as a surprise, as the two smaller groups participating in the survey (the ICA and ECREA groups) are part of dedicated "communication" associations. However, when taking into account that the much larger partner association, at least in terms of direct reach to digital games researchers, was DiGRA, and that previous works (Williams, 2005, p. 2) noted a dominance of "humanistic work" in that association, the findings may be indicative of a change in the composition of the field, and, indeed, a disciplinary reshaping with a strong centripetal force toward social sciences. It is also notable that there is only a small group of engineers and computer scientists in the field, which supports our notion of a differentiation of technologically oriented scientists (with their debates taking place in computer sciences and engineering associations, such as ACM SIG or IEEE, and in industry-related groups). The large majority of the academics in the survey also consider themselves dedicated games researchers, and they typically play games themselves (similar to adult populations in Western societies in general; see Quandt et al., 2014).

Overall, the support for a socially beneficial effect of games is high, whereas the attitudes toward harmful effects of digital games are more mixed. The latter result, however, should be interpreted somewhat tentatively due to moderate measurement reliability for the outcome measure. Still, our findings contradict the claim of a "broad consensus" (Bushman et al., 2014) regarding the negative impact of digital games, as the opinions expressed by the participants in this study are not widely supportive of 
Table 4 Impact of Sociodemographics, Academic Background, Gaming Behavior, and Self-Perception of Digital Games Researchers on Attitude Toward Harmful Effects of Digital Games $(n=483)$

\begin{tabular}{|c|c|c|c|c|c|}
\hline Source of Variance & $\begin{array}{l}\text { Sum of } \\
\text { Square }\end{array}$ & $d f$ & $F$ & $\eta^{2}$ & $d$ \\
\hline \multicolumn{6}{|l|}{ Sociodemographics } \\
\hline Age (years) & 0.355 & 1 & 0.648 & 0.001 & 0.063 \\
\hline Gender & 0.042 & 1 & 0.077 & 0.000 & 0.025 \\
\hline \multicolumn{6}{|l|}{ Academic background } \\
\hline Teaching digital games (yes) & 1.942 & 1 & 3.550 & 0.007 & 0.168 \\
\hline Discipline & 11.615 & 10 & $2.124^{\star}$ & 0.043 & 0.424 \\
\hline \multicolumn{6}{|l|}{ Gaming behavior } \\
\hline Time gaming per day (minutes) & 0.433 & 1 & 0.792 & 0.002 & 0.090 \\
\hline Never actively played digital games (yes) & 2.327 & 1 & $4.254^{\star}$ & 0.009 & 0.196 \\
\hline \multicolumn{6}{|l|}{ Self-perception } \\
\hline I see myself as a digital games researcher & 1.812 & 1 & 3.313 & 0.007 & 0.168 \\
\hline I see myself as a gamer & 0.335 & 1 & 0.613 & 0.001 & 0.063 \\
\hline Error & 254.314 & 465 & & & \\
\hline
\end{tabular}

Note: $R^{2}=.070$.

${ }^{*} p \leq .05 .{ }^{* *} p \leq .01$.

such a position. This may be due to a number of reasons. First of all, there is a clear difference in scope and manner of inquiry in this study compared with that by Bushman and colleagues. They were primarily interested in the potential existence of an effect of violent games on aggressive behavior, without reference to its size or importance, as illustrated by the item used "Violent video games can increase aggressive behavior in children," whereas our study focused on its societal impact as illustrated by the following item "The effects of digital games on aggression behavior are a problem for society." When we look at another, more similar specific item in our study, "Digital games can have harmful effects on their users," we find a result more comparable to that of Bushman and colleagues. Even there, a difference still exists, however, which is probably due to a difference in sampling and hence the population about which claims can be made. Although this survey was distributed among digital games researchers, Bushman and colleagues sampled effects researchers from respective groups within APA and ICA (i.e., their findings do not depict the "inside" view of the field, as outlined in our model, but rather the perspective of other, more central disciplinary groups on the phenomenon). Nonetheless, it should be noted that our sample is relatively homogeneous (especially when compared with our initial expectations) - so this, again, could be interpreted as a consensus within this respondent group. This may indeed be an effect of the identification with "digital games researchers" as a reference group (following Merton's theoretical argument). Either way, the findings counter the notion of a prevailing negative view of digital games' effects among researchers working on the topic. 
We also found that the belief in beneficial effects does not necessarily go hand-in-hand with disbelief in the existence of potential negative effects - the statistical analyses found these to be independent constructs that were not correlated at all. Detailed analysis of the determinants of the two constructs revealed an even more differentiated picture of the state of the field: There are significant differences between various formal and informal groups. These groups may shape the further development of the field, by framing the new knowledge according to their respective viewpoints. The strongest group effects could be found for disciplinary background: Depending on the "mother discipline" of the researchers (i.e., reflecting their personal disciplinary upbringing), viewpoints differ notably. Again, the effects are not uniform for the two constructs under analysis: For example, the educational scientists were most positive when it came to the beneficial effects, while they were among the more critical scholars when it came to negative effects (this may be indicative of more "pronounced" opinions among this group of scholars). On the other hand, researchers from sociology and the humanities were more critical for both constructs, which might imply a skeptical attitude toward the effects hypothesis in general. Media and communication scholars agreed moderately, and surprisingly, psychologists had an above average, positive view of digital games, more strongly believing in beneficial effects, and less in harmful effects. These findings concerning the psychologists in the sample seem to oppose a general perception of effects researchers being overly negative toward digital games. This may, for example, be explained by empirical work showing less pronounced effects than anticipated, leading to a more positive view. Another explanation may lie in the different reference groups (based on Merton's argument): Although they perceive themselves as psychologists, they also perceive themselves as game scholars, that is, psychologists in DGR. Now other effects researchers, who are more skeptical, may not be related to the associations supporting the survey, and they may subsume themselves under another field that is much more critical toward digital games (i.e. general media effects research in psychology). The differentiation between these two groups of psychologists may explain more positive viewpoints of psychologists in DGR as they try to distance themselves from the "other" psychologists - but this assumption cannot be tested on the basis of this study (see Limitations).

There were some other notable but small effects - or a lack thereof: We found no gender effect - male and female researchers do not differ in their perceptions. However, we obtained an age effect, but in the opposite direction of what we expected: Older researchers were more strongly believing in beneficial effects of digital games. Seniority, then, was not a negative factor, in contrast to a hypothesized age-related skepticism (see above).

Furthermore, self-perceptions as digital games researcher and as a gamer also lead to a stronger belief in beneficial effects but have less influence on the belief in negative effects. Thus, it may be argued that scholars who identify strongly with the field and the medium are somewhat more hopeful regarding their subject area's societal potential but not less critical of its potential downsides. 
Notably, playing time did not have an impact on perceptions. The only significant effect of personal gameplay behavior on perceptions could be found for harmful effects: Researchers who have absolutely no personal experience with using the medium believe more strongly in its harmful qualities. This is an interesting fact, as it means that researchers who never experienced game effects personally more strongly believe that games can negatively impact others. This is corroborated by findings of Ivory and Kalyanaraman (2009); Przybylski (2014); and Royse, Lee, Baajanjav, Hopsons, and Consalvo (2007), which suggest that not being exposed to games predicts stronger beliefs about negative effects on others, stronger support for censorship, and stronger belief in games being antisocial.

Overall, these findings depict a field that is different from what has been reported in previous literature, and that is probably also different from public expectations. Still, the viewpoints are differentiated, and despite a notable homogeneity of the data, there are significant differences between some of the subgroups in the field. These formal and informal groups will certainly influence the further development of DGR, but the deviations between the groups are not insurmountable to the extent that they would support the notion of a "clash of camps."

\section{Limitations}

This study has several limitations, some of them by design. First of all, the study took a deliberate "inside" perspective by focusing on researchers that were part of or connected to the working groups in ICA, ECREA, or DiGRA. Naturally, this circumscribes the field and its boundaries in a specific way, through connections to some formal groups. In principle, an alternative solution would be a survey among all scholars in the broader disciplines, also reaching those who do not assign themselves to formal groups but still work in the field. For practical research reasons, this is probably not feasible, and additionally, a full disciplinary survey may also have led to a much lower response rate.

Furthermore, the sample itself was more homogeneous than expected. The absence of radically different camps is a major finding of this study. A much larger sample would be needed to identify more distinct subgroups within the homogeneous community of games researchers.

By design, the study was limited by the number of items of the questionnaire. The current set of questions had the aim to provide some unique base information on the state of the field, while keeping the willingness to participate among scholars high. The length of the questionnaire was balanced against the utility of including more detailed scales and additional items. Naturally, a follow-up study may focus on more dedicated questions and scales based on some of the initial questions answered by this study.

Finally, this study is a mere snapshot, with next to no preexisting data for comparison. As noted above, previous works on the constitution of the field were primarily based on anecdotal evidence and individual viewpoints, so any conjectures on the development of the field were just based on plausible assumptions. However, future 
studies may take our initial exploration as a starting point for time-based analyses that would allow for insights into the development of the field.

\section{Note}

1 In this study, we focus on the development of digital games as a research topic within the social sciences and humanities, and communication studies in particular. It has been noted that communication studies itself may indeed be a multidisciplinary field, or a discipline with multiple influences from other neighboring disciplines, ranging from psychology to sociology to pedagogics and more (Sjøvaag \& Moe, 2009; see also the influential 1983 special issue of Journal of Communication, "Ferment in the field"). So we also conceptualize digital games research within communication studies as a multidisciplinary field, but with firm roots in social sciences and humanities. Some researchers from other disciplines, most notably human-computer interaction, engineering and computer sciences, are active in that field as well. However, we do not feel we can speak for the core of these other disciplines and restrict our claims to the three organizations involved in this study.

\section{References}

Aarseth, E. (2001, July). Computer game studies, year one. Game Studies, 1(1). Retrieved from http://www.gamestudies.org/0101/editorial.html

Anderson, C. A., Shibuya, A., Ihori, N., Swing, E. L., Bushman, B. J., Sakamoto, A., Rothstein, H.R., \& Saleem, M. (2010). Violent video game effects on aggression, empathy, and prosocial behavior in eastern and western countries: A meta-analytic review. Psychological Bulletin, 136(2), 151 - 173. doi:10.1037/a0018251.

Barber, B. (1961). Resistance by scientists to scientific discovery. Science, 134(3479), $596-602$.

Baym, N. K. (2005). Introduction: Internet research as it isn't, is, could be, and should be. The Information Society, 21(4), 229-232.

Berger, T., \& Luckmann, T. (1966). The social construction of reality: A treatise in the sociology of knowledge. Garden City, NY: Anchor Books.

Bushman, B. J., Gollwitzer, M., \& Cruz, C. (2014). There is broad consensus: Media researchers agree that violent media increase aggression in children, and pediatricians and parents concur. Psychology of Popular Media Culture, 4(3), 200-214. doi:10.1037/ ppm0000046.

Cohen, J. (1988). Statistical power analysis for the behavioral sciences. Hillsdale, NJ: Erlbaum.

Connolly, T. M., Boyle, E. A., MacArthur, E., Hainey, T., \& Boyle, J. M. (2012). A systematic literature review of empirical evidence on computer games and serious games. Computers \& Education, 59(2), 661-686.

Consortium of Scholars. (2013). Scholar's open statement to the APA task force on violent media. Retrieved from http://www.christopherjferguson.com/APA\%20Task\%20Force\% 20Comment1.pdf

De Grove, F., \& Van Looy, J. (2015). How to be a gamer! Exploring personal and social indicators of gamer identity. Journal of Computer-Mediated Communication, 20(3), 346-361. doi:10.1111/jcc4.12114. 
Durkheim, E., \& Mauss, M. (1901 - 1902). De quelques formes de classification - contribution à l'étude des représentations collectives. L'année Sociologique, 6, 1-72.

Entertainment Software Association. (2014). Essential facts about the computer and video game industry. 2014 sales, demographic and usage data. Retrieved from http://www.theesa.com/wp-content/uploads/2014/10/ESA_EF_2014.pdf

Ferguson, C. J. (2007). The good, the bad and the ugly: A meta-analytic review of positive and negative effects of violent video games. Psychiatric Quarterly, 78(4), 309-316. doi:10.1007/s11126-007-9056-9.

Field, A. (2009). Discovering statistics using SPSS. London, England: Sage.

Gingras, Y., Larivière, V., Macaluso, B., \& Robitaille, J.-P. (2008). The effects of aging on researchers' publication and citation patterns. PLoS One, 3(12), e4048. doi:10.1371/journal.pone.0004048.

Griffiths, M. D., Davies, M. N. O., \& Chappell, D. (2003). Breaking the stereotype: The case of online gaming. CyberPsychology \& Behavior, 6(1), 81-91. doi:10.1089/10949310 3321167992.

Hyman, H. H. (1942). The psychology of status. Archives of Psychology, 269, 5-94.

Ivory, J. D. (2013). Video games as a multifaceted medium: A review of quantitative social science research on video games and a typology of video game research approaches. Review of Communication Research, 1(2), 31 -69. doi:10.12840/issn.2255-4165_ 2013_01.01_002.

Ivory, J. D., \& Kalyanaraman, S. (2009). Video games make people violent - well, maybe not that game: Effects of content and person abstraction on perceptions of violent video games' effects and support of censorship. Communication Reports, 22(1), 1-12. doi:10.1080/08934210902798536.

Kuhn, T. (1962). The structure of scientific revolutions. Chicago, IL: University of Chicago Press.

Lemaine, G., Macleod, R., Mulkay, M., \& Weingart, P. (1976). Introduction: Problems in the emergence of new disciplines. In G. Lemaine, R. Macleod, M. Mulkay, \& P. Weingart (Eds.), Perspectives on the emergence of scientific disciplines (pp. 1-23). The Hague: Mouton.

Mannheim, K. (1929). Ideologie und utopie. Bonn, Germany: Cohen.

Mäyrä, F. (2008). An introduction to game studies. London, England: Sage.

Mäyrä, F., Van Looy, J., \& Quandt, T. (2013). Disciplinary identity of game scholars: An outline. In Proceedings of DiGRA 2013. Retrieved from http://hdl.handle.net/ 1854/LU-4225122

Meade, A. W., \& Crais, S. B. (2012). Identifying careless responses in survey data. Psychological Methods, 17(3), 437-455.

Merton, R. K. (1957). Social theory and social structure. Glencoe, IL: Free Press.

Merton, R. K. (1968). The matthew effect in science. Science, 159(3810), 56-63. doi:10.1126/ science.159.3810.56.

Merton, R. K., \& Zuckerman, H. (1973). Institutionalized patterns of evaluation in science. In N. Storer (Ed.), The sociology of science: Theoretical and empirical investigations (pp. 460 -496). Chicago, IL: University of Chicago Press.

Mortensen, T. (2009). The art and study of computer games. New York: Peter Lang. 
Pearce, C. (2005). Theory wars: An argument against arguments in the so-called ludology/narratology debate. In Proceedings of DiGRA 2005. Retrieved from http://www.digra.org/wp-content/uploads/digital-library/06278.03452.pdf

Pollard Sacks, D., Bushman, B. J., \& Anderson, C. A. (2011). Do violent video games harm children? Comparing the scientific amicus curiae "experts" in Brown v. Entertainment Merchants Association. Northwestern University Law Review Colloquy, 106, 1-12.

Polsby, N. W. (1998). Social science and scientific change: A note on Thomas S. Kuhn's contribution. Annual Review of Political Science, 1, 199-210.

Przybylski, A. K. (2014). Who believes electronic games cause real world aggression? Cyberpsychology, Behavior and Social Networking, 17(4), 228-234. doi:10.1089/cyber.2013.0245.

Quandt, T., Chen, V., Mäyrä, F., \& Van Looy, J. (2014). (Multiplayer) Gaming around the globe? A comparison of gamer surveys in four countries. In T. Quandt \& S. Kröger (Eds.), Multiplayer: The social aspects of digital gaming (pp. 23-46). London, England: Routledge.

Royse, P., Lee, J., Baajanjav, U., Hopsons, M., \& Consalvo, M. (2007). Women and games: Technologies of the gendered self. New Media \& Society, 9(4), 555-576.

Shaw, A. (2012). Do you identify as a gamer? Gender, race, sexuality, and gamer identity. New Media \& Society, 14(1), 28-44. doi:10.1177/1461444811410394.

Sherry, J. L. (2001). The effects of violent video games on aggression: A meta-analysis. Human Communication Research, 27(3), 409-431. doi:10.1111/j.1468-2958.2001.tb00787.x.

Šidák, Z. (1967). Rectangular confidence regions for the means of multivariate normal distributions. Journal of the American Statistical Association, 62(318), 626-633. doi:10.1080/01621459.1967.10482935.

Sjøvaag, H., \& Moe, H. (2009). From fermentation to maturity? Reflections on media and communication studies: An interview with Todd Gitlin, Jostein Gripsrud \& Michael Schudson. International Journal of Communication, 3, 130-139.

Turner, J. C., Oakes, P. J., Haslam, S. A., \& McGarty, C. M. (1994). Self and collective: Cognition and social context. Personality and Social Psychology Bulletin, 20, 454-463.

Van Looy, J. (2010). Understanding computer game culture: The cultural shaping of a new medium. Saarbrücken, Germany: Lambert Academic.

Velicer, W. (1976). Determining the number of components from the matrix of partial correlations. Psychometrika, 41(3), 321-327.

Williams, D. (2005). Bridging the methodological divide in game research. Simulation \& Gaming, 36(4), 447-463. doi:10.1177/1046878105282275.

Zuckerman, H., \& Merton, R. K. (1973). Age, aging and age structure in science. In R. K. Merton (Ed.), The sociology of science (pp. 493-560). Chicago, IL: Chicago University Press. 